\title{
Murine induced pluripotent stem cells can be derived from and differentiate into natural killer T cells
}

\author{
Hiroshi Watarai, ${ }^{1,2}$ Shin-ichiro Fujii,, ${ }^{1}$ Daisuke Yamada, ${ }^{1,2}$ Andrei Rybouchkin,, Sakura Sakata, ${ }^{1}$ \\ Yuko Nagata, ${ }^{1}$ Midori lida-Kobayashi, ${ }^{1,2}$ Etsuko Sekine-Kondo, ${ }^{1}$ Kanako Shimizu, ${ }^{1}$ Yohei Shozaki, ${ }^{1}$ \\ Jafar Sharif, ${ }^{1}$ Masashi Matsuda, ${ }^{1}$ Shinobu Mochiduki, ${ }^{1}$ Takanori Hasegawa, ${ }^{1}$ Genta Kitahara, ${ }^{1}$ \\ Takaho A. Endo, ${ }^{3}$ Tetsuro Toyoda, ${ }^{3}$ Osamu Ohara, ${ }^{1}$ Ken-ichi Harigaya, ${ }^{4}$ \\ Haruhiko Koseki, ${ }^{1,2}$ and Masaru Taniguchi'
}
${ }^{1}$ RIKEN Research Center for Allergy and Immunology, Yokohama, Japan. ${ }^{2}$ Core Research for Evolutional Science and Technology (CREST), Japan Science and Technology Corporation (JST), Tokyo, Japan. ${ }^{3}$ RIKEN BASE, Yokohama, Japan. ${ }^{4}$ Department of Pathology, Graduate School of Medicine, Chiba University, Chiba, Japan.

\begin{abstract}
NKT cells demonstrate antitumor activity when activated to produce Th1 cytokines by DCs loaded with $\alpha$-galactosylceramide, the prototypic NKT cell-activating glycolipid antigen. However, most patients do not have sufficient numbers of NKT cells to induce an effective immune response in this context, indicating a need for a source of NKT cells that could be used to supplement the endogenous cell population. Induced pluripotent stem cells (iPSCs) hold tremendous potential for cell-replacement therapy, but whether it is possible to generate functionally competent NKT cells from iPSCs has not been rigorously assessed. In this study, we successfully derived iPSCs both from embryonic fibroblasts from mice harboring functional NKT cell-specific rearranged $T$ cell receptor loci in the germline and from splenic NKT cells from WT adult mice. These iPSCs could be differentiated into NKT cells in vitro and secreted large amounts of the Th1 cytokine IFN- $\gamma$. Importantly, iPSC-derived NKT cells recapitulated the known adjuvant effects of natural NKT cells and suppressed tumor growth in vivo. These studies demonstrate the feasibility of expanding functionally competent NKT cells via an iPSC phase, an approach that may be adapted for NKT cell-targeted therapy in humans.
\end{abstract}

\section{Introduction}

NKT cells are characterized by their expression of an invariant TCR $\alpha$ chain encoded by $V \alpha 14-J \alpha 18$ in mice (1) and by $V \alpha 24$ Ja18 in humans $(2,3)$. NKT cells recognize glycolipid antigens and can be efficiently activated through recognition of $\alpha$-galactosylceramide $(\alpha$-GalCer) in the context of CD1d, a monomorphic MHC class I-like molecule (4). Although activated NKT cells produce both Th1 and Th2 cytokines, they mediate strong adjuvant activity essential for protective responses against tumors (5-7) and pathogens through their production of Th1 cytokines.

Based on these observations, $\alpha$-GalCer can be used as a drug for clinical applications. Several clinical trials with injection of $5 \times 10^{6}$ $\alpha$-GalCer-pulsed DCs have been carried out in patients with cancer, including colon cancer $(8,9)$, multiple myeloma, anal cancer, and renal cell cancer (10). Although no clear tumor reduction was detected, tumor markers were significantly decreased.

A more extensive phase I/IIa clinical trial of $\alpha$-GalCer-loaded DC therapy (total $4 \times 10^{9}$ cells per person in 4 consecutive injections at 1 week intervals) for advanced lung cancer patients (stages IIIB, IV, and recurrent) was launched. Encouragingly, this trial validated the antitumor effects of NKT cells in humans, since $60 \%$ of 17 enrolled patients with only a primary treatment showed a prolonged median survival time (MST) of 31.9 months

Authorship note: Hiroshi Watarai and Shin-ichiro Fujii contributed equally to this work.

Conflict of interest: The authors have declared that no conflict of interest exists. Citation for this article: J Clin Invest. 2010;120(7):2610-2618. doi:10.1172/JCI42027. without tumor progression and metastasis compared with the MST of 4.6 months under the best supportive care (11). However, despite its clear antitumor activity, two-thirds of patients were not eligible for NKT cell-targeted therapy because they no longer had sufficient NKT cells (less than 10 cells $/ \mathrm{ml}$ of blood) (12). These observations underscore the urgent need to establish an efficient method to supplement NKT cells.

Results from our previous study, in which we used embryonic stem cells (ESC) derived from NKT cell-cloned mice (hereafter called the NKT mouse) established by nuclear transfer from C57BL/6 (B6) hepatic NKT cells, had already suggested that these cells could give rise to NKT cells both in vivo and in vitro (13). Since induced pluripotent stem cells (iPSCs) and ESCs are functionally equivalent in many respects, these observations suggested the feasibility of the clinical potential of iPSCs for NKT cell-targeted adjuvant therapy (14-16). Moreover, it is important to mention that iPSCs rather than ESCs are more feasible in the clinical setting because embryos or donor oocytes are not required for generation of iPSCs. In addition, iPSCs are fully syngeneic, while ESCs, even if introduced with patient cell nucleus, carrying oocyte-derived mitochondrial maternal antigens may cause problems in generating allogeneic immune responses. The present study has been designed to examine the feasibility of ex vivo generation of functionally competent NKT cells from iPSCs. Here, we have addressed 2 specific issues: (a) generation of iPSCs from mature NKT cells and their in vitro generation of functional NKT cells, and (b) investigation of the adjuvant activity of iPSC-derived NKT cells transferred in vivo and their clinical potential in a mouse model. 
A
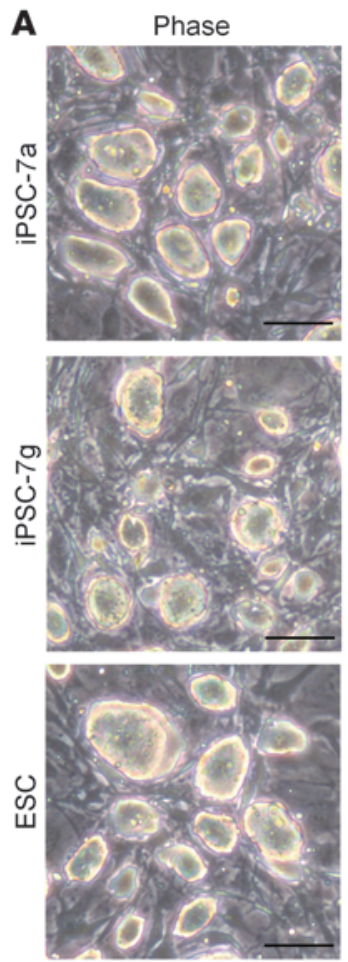

SSEA1 DAPI
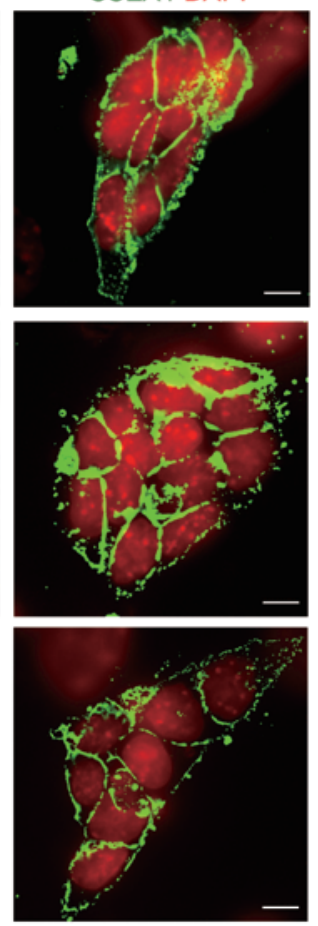

Oct3/4 DAPI
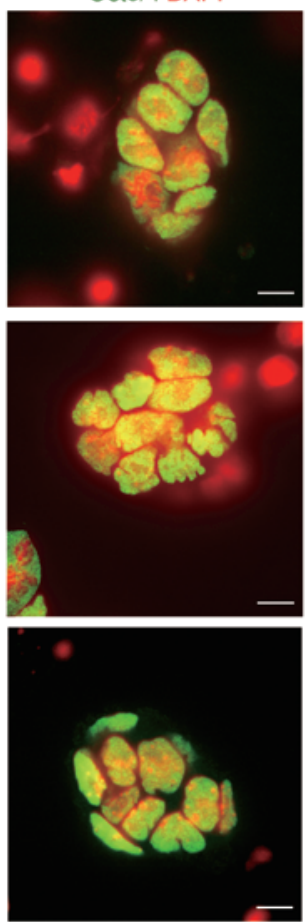

Nanog DAPI
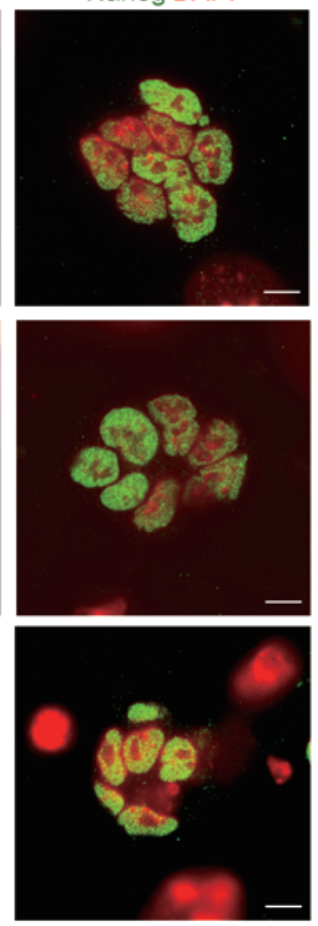

B

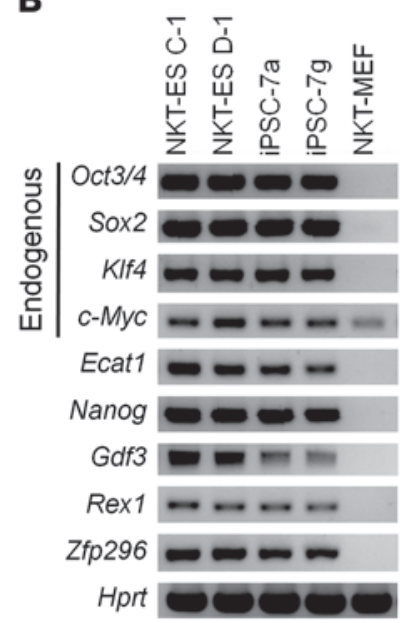

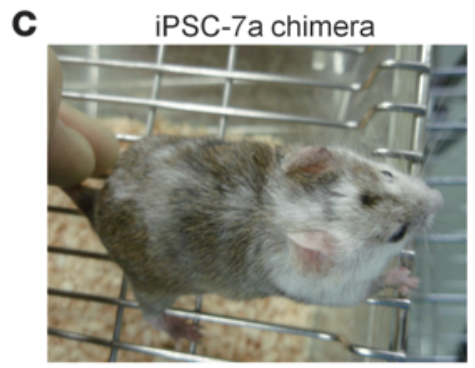
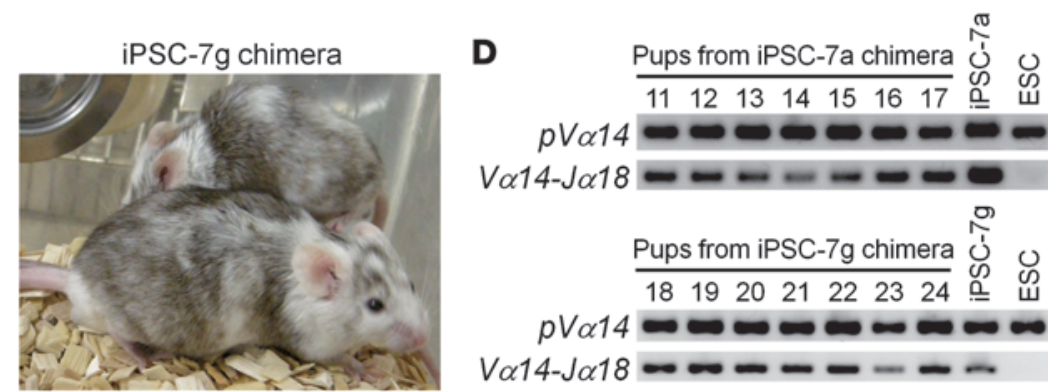

$p V \alpha 14=000000$ V $\alpha 14-J \alpha 18-----\infty-6$

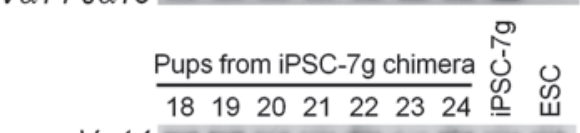

$p V \alpha 14=-\infty 0000$

V $\alpha 14-J \alpha 18-\infty-\infty-\cdots$

\section{Figure 1}

Generation of iPSCs harboring NKT cell-specific rearranged T cell receptor loci. (A) Colony shape and the expression of stage-specific embryo antigen 1 (SSEA1), Octamer-binding protein 3/4 (Oct3/4), and Nanog in the iPSC-7a and iPSC-7g cells. ESCs are shown as a reference. Scale bars: $100 \mu \mathrm{m}$ (phase); $10 \mu \mathrm{m}$ (immunofluorescence). (B) Expression of ESC marker genes in iPSC-7a and iPSC-7g cells. Results for RT-PCR analyses are shown. NKT-ES C-1 and NKT-ES D-1 were used as positive controls, and NKT-MEF was used as a negative control. (C) External views of chimeras generated by injecting iPSC-7a and iPSC-7g cells into BALB/c blastocysts. (D) Transmission of the rearranged Tcra chain locus to the offspring of chimeras, which is characteristic of iPSC-7a (upper panel) and iPSC-7g cells (lower panel).

\section{Results}

Generation of iPSCs harboring NKT cell-specific rearranged T cell receptor loci. Mouse embryonic fibroblasts obtained from the NKT mice (NKT-MEF) that had been established from ESCs generated by NKT cell nuclear transfer (NKT-ES) (13) were reprogrammed to generate iPSCs using a conventional protocol with retroviral vectors (14). Eight ESC-like colonies were selected and expanded in ES medium. We found that most of the lines stably exhibited ESC-like morphology and selected lines, iPSC-7a and iPSC-7g, for further analysis to verify that they were indeed iPSCs. We analyzed their expression of molecular markers that define iPSCs and determined the degree of reprogramming and their ability to generate teratomas and chimeras.

Immunofluorescence analysis revealed that the expression of SSEA1, Oct3/4, and Nanog in both the iPSC-7a and iPSC-7g lines was similar to that in the control ESCs (Figure 1A). We also detected the expression of endogenous Oct3/4, Sox2, Klf4, Nanog, Ecat1, Gdf3, Rex1, and Zfp296 by RT-PCR analysis (Figure 1B). Consistent with the reactivated expression of Oct3/4 and Nanog in the iPSC-7a and iPSC-7g cells, DNA methylation patterns at these gene loci were similar to ESCs and significantly reduced in comparison with the parental NKT-MEF (Supplemental Figure 1; supplemental material available online with this article; doi:10.1172/JCI42027DS1).

We went on to compare the gene expression profiles of the iPSC$7 \mathrm{a}$ and iPSC-7g cells against NKT-ES and NKT-MEF. The genomewide expression profile of the iPSC-7a cells showed a much higher correlation coefficient to NKT-ES C-1 than to the parental NKTMEF (Supplemental Figure 2A). Cluster analysis placed the iPSC-7a and iPSC-7g cells in the NKT-ES (C-1 and D-1) or ESCs but not the NKT-MEF, iPSC-derived NKT cells (7a-NKT and 7g-NKT) nor 
A
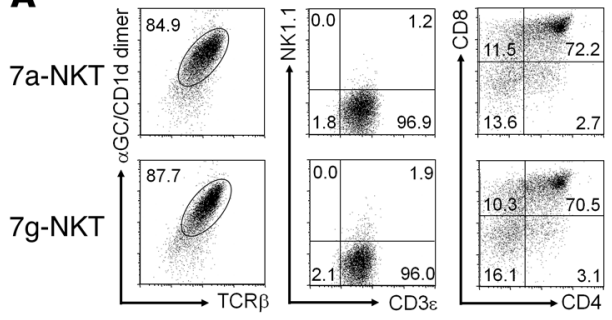

B

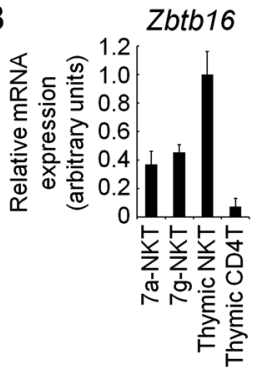

Sh2d1a

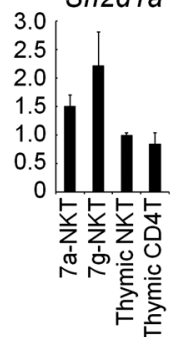

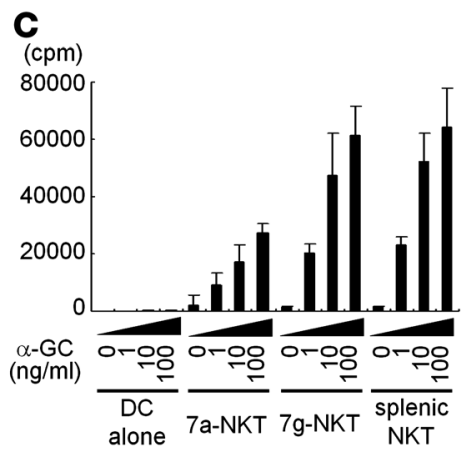
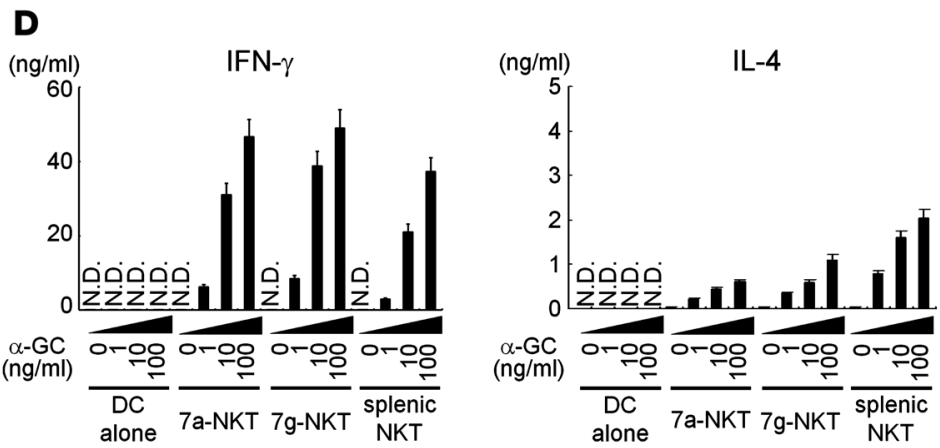

Figure 2

In vitro generation of NKT cells from iPSCs in the 25-day culture system. (A) Expression of cell surface markers on iPSC-derived NKT cells (7a-NKT and 7g-NKT). 7a-NKT and 7g-NKT cells generated in vitro were gated as the $\alpha$-GalCer/CD1d dimer+TCR- $\beta^{+}$population and further analyzed for the expression of the indicated markers, NK1.1 versus CD3E and CD4 versus CD8. Numbers show percentage of each gate. (B) Quantitative PCR analysis. Indicated genes in iPSC-derived NKT cells and FACS-sorted thymic NKT and CD4 T cells were analyzed for mRNA levels by quantitative real-time PCR $\left(2 \times 10^{3}\right.$ cells/sample). (C and D) Proliferative response (C) and cytokine production (D) of iPSC-derived NKT cells upon stimulation with $\alpha$-GalCer. The 7a-NKT, $7 \mathrm{~g}-\mathrm{NKT}$, or WT splenic NKT cells (106/ml) were cocultured with bone marrow-derived DCs $\left(10^{5} / \mathrm{ml}\right)$ in the presence of the indicated dose of $\alpha$-GalCer $(0,1,10,100 \mathrm{ng} / \mathrm{ml})$. Mean \pm SD of triplicate wells is shown. One representative experiment of 3 is shown.

splenic NKT cell (NKT 1 and NKT 2) cluster (Supplemental Figure 2 , B and C). Therefore, the iPSC-7a and iPSC-7g cells were sufficiently reprogrammed. Furthermore, we tested the ability of these cells to form teratomas in nude mice and produce germline chimeras upon blastocyst injection. Both iPSC-7a and iPSC-7g cells formed teratomas with 3 germ layers upon s.c. injection into nude mice (D. Yamada and H. Koseki, unpublished observations), and when injected into BALB/c blastocysts, they generated 7 and 3 coat color chimeras, respectively (Figure $1 C$ ). The male chimeras tested were found to be germline chimeras that transmitted the rearranged Tcra loci to their offspring (Figure 1D). We thus concluded the iPSC-7a and iPSC-7g lines satisfied all the known criteria for iPSCs.

In vitro generation of NKT cells from iPSCs in the 20-day culture system. We first attempted to investigate the potential of NKT cell development from iPSC-7a and iPSC-7g in the 20-day culture system used for analysis of ESCs described previously (Supplemental Figure 3A) (13). Similar to ESC-derived NKT cells, iPSCderived NKT cells mainly produced IL-4 on OP9/Dll-1 culture for 20 days, while mainly producing IFN- $\gamma$ in the switching culture on OP9/control starting at day 14 (Supplemental Figure 3B), indicating that iPSCs are potentially the same as ESCs. Moreover, the yield of NKT cells from the Notch-dependent conditions was $3 \times 10^{6}$ from $1 \times 10^{5}$ iPSCs (30 times), while those from Notch-independent conditions were only $3 \times 10^{5}$ ( 3 times). Thus, the culture conditions with or without Notch signaling reflected the proliferation activity of iPSC-derived NKT cells such as ESCderived NKT cells in vitro (Supplemental Figure 3C).
The 25-day culture system efficient for generation of iPSC-derived NKT cells producing large amounts of IFN- $\gamma$. It is important to obtain a high yield of NKT cells with the desired function for the establishment of NKT cell therapy. For this purpose, we attempted to develop a new 25-day culture system efficiently to generate functional NKT cells from the iPSC-7a and iPSC-7g cells in vitro. Since proliferative activity is totally depend on Notch signaling (Supplemental Figure 3D), iPSCs were cocultured on OP9/Dll-1 in the presence of various cytokine combinations to manipulate NKT cell function (Supplemental Figure 4A). The addition of IL-15 at culture day 20 for 5 days suppressed the proliferative function of NKT cells (Supplemental Figure 4B), while IL-2 promoted NKT cell expansion but suppressed IFN- $\gamma$ production upon stimulation with $\alpha$-GalCer (Supplemental Figure 4B). In contrast with the above cytokines, the addition of both IL-7 and Flt-3 ligand (Flt3L) at the culture day 20 for 5 days yielded the highest number of NKT cells and also the highest amounts of IFN- $\gamma$ production (Supplemental Figure 4B), which are equivalent to those by splenic NKT cells (see Figure 2D). Under these conditions, $1 \times 10^{5}$ iPSCs gave rise to $3 \times 10^{7}$ iPSC-derived NKT cells at culture day 25 (Supplemental Figure 4C) that expressed TCR- $\beta$ on their surface and bound $\alpha$-GalCer-loaded soluble CD1d dimer, which are specifically recognized by the NKT-specific TCR (Figure 2A). The iPSC-derived NKT cells (7a-NKT and 7g-NKT) did not express NK1.1 (Figure 2A). Moreover, a significant fraction of the 7a-NKT and $7 \mathrm{~g}-\mathrm{NKT}$ cells were doubly positive for CD4 and CD8 (Figure 2A). Therefore, the iPSC-derived NKT cells generated in this 25-day culture 
system were more similar to immature $\mathrm{CD} 4^{+} \mathrm{CD} 8^{+}$(double-positive [DP]) thymocytes than to mature liver NKT cells. Moreover, they were CD24hi, CD44lo/int $, \mathrm{CD} 62^{\mathrm{hi}}, \mathrm{CD} 69^{+}, \mathrm{CD} 122^{\mathrm{lo} /-}$, and NKG2D-, which phenotypically resemble the earliest thymic NKT cells, so-called stage 0 NKT cells (Supplemental Figure 5). The iPSC-derived NKT cells expressed significant levels of Zbtb16 (also known as PLZF) $(17,18)$ and also Sh2d1a (also known as SAP) $(19$, 20) compared with those of thymic CD4 T cells, indicating that iPSC-derived cells are phenotypically immature and likely to be typical NKT cells, but not conventional T cells (Figure 2B).

To further investigate functional activity of iPSC-derived NKT cells, proliferative responses and cytokine production were investigated upon stimulation with $\alpha$-GalCer-pulsed DCs (Figure 2, Cand D). The iPSC-derived NKT cells from both iPSC-7 $\mathrm{a}$ and iPSC-7g proliferated similarly to splenic NKT cells (Figure 2C). The cytokines, such as IFN- $\gamma$, IL-4, IL-5, IL-10, and IL-13, were also produced by iPSC-derived NKT cells at levels similar to those of splenic NKT cells (Figure 2D and Supplemental Figure 6). Taken together, these observations indicated that the iPSC-derived NKT cells possess functional properties similar to those of NKT cells. It is also important to mention that, as opposed to the 20-day culture system used for iPSC-derived (Supplemental Figure 3) or ES-derived NKT cell development (13), the 25-day culture system yielded 10 times the number of NKT cells and produced 5 times the amount of IFN- $\gamma$ (Figure 2 and Supplemental Figure 4).

Adjuvant activity of $i$ PSC-derived NKT cells. We then addressed whether iPSC-derived NKT cells (7a-NKT or 7g-NKT) can exert adjuvant activity in vivo in mice. We and others have previously developed an experimental model to evaluate the adjuvant activity of activated NKT cells (21-24). In this model, we observed significant enhancement of antigen-specific CD8 $\mathrm{T}$ cell priming by coadministration of OVA-loaded dying spleen cells from $T A P^{-1-}$ mice $\left(2 \times 10^{7} /\right.$ mouse $)$ with $2 \mu \mathrm{g} \alpha$-GalCer (TOG) in an NKT cell-dependent manner (Supplemental Figure 7$)(23,24)$. We first determined whether iPSCderived NKT cells could repopulate in the recipient tissues after adoptive transfer into J $\alpha 18^{-/-}$mice, which lack endogenous NKT cells. A considerable number of the 7a-NKT and 7g-NKT cells were detectable in the liver of $J \alpha 18^{-/-}$mice 1 and 2 weeks after the transfer (Figure 3A). These cell numbers are almost similar to those of WT mature liver NKT cells transferred into $J \alpha 18^{-/-}$mice. Interestingly, 7a-NKT and 7g-NKT cells detected in the liver were CD24lo, CD44hi, $\mathrm{CD}_{62} \mathrm{~L}^{\text {lo }}, \mathrm{CD} 9^{+}, \mathrm{CD} 122^{+}$, and $\mathrm{NKG}_{2} \mathrm{D}^{+}$, phenotypically similar to mature liver NKT cells in WT mice (Supplemental Figure 8), indicating the maturation of their phenotypes in vivo. To test the ability of IFN- $\gamma$ production from iPSC-derived NKT cells upon antigen stimulation, the mice that received the 7a-NKT or $7 \mathrm{~g}-\mathrm{NKT}$ cells were injected with $\alpha$-GalCer i.v. $\alpha$-GalCer administration led to the significant expansion (0.3\%-0.4\% [Figure 3A] vs. 4.9\%-5.7\% [Figure $3 \mathrm{~B}]$ ) of $7 \mathrm{a}-\mathrm{NKT}$ and $7 \mathrm{~g}-\mathrm{NKT}$ cells with downmodulated TCR- $\beta$ expression (Figure 3B vs. Figure $3 \mathrm{~A}$ ) and significant IFN- $\gamma$ production (Figure 3B), indicating the in vivo activation of iPSC-derived NKT cells. Moreover, we observed in vivo bystander adjuvant effects on NK $1.1^{+}$TCR- $\beta^{-}$cells after activation of iPSC-derived NKT cells in terms of their significant IFN- $\gamma$ production (Figure $3 \mathrm{C}$ ).

We further investigated the bystander adjuvant activity by the iPSC-derived NKT cells on CD8 T cells in vivo, in which the mice transferred with the 7a-NKT or 7g-NKT cells were immunized with TOG and examined for the extent of induction of OVA-specific, IFN- $\gamma$-producing CD8 T cells. We indeed observed a significant increase in the number of these CD8 T cells (10-50 times increases over the control group) (Figure 3, D and E). Thus, the iPSC-derived NKT cells were shown to function as a cellular adjuvant for both innate and adaptive immune responses.

We next adoptively transferred the 7a-NKT or $7 \mathrm{~g}$-NKT cells into $J \alpha 18^{-/-}$mice to test for their acquisition of antigen-specific antitumor activity. Seven days after the adoptive transfer, mice were primed with TOG, challenged with EL4, a B6-derived thymoma line, or with the OVA-expressing EL4 subline EG7 $\left(2 \times 10^{5}\right.$ cells injected s.c.), and then observed for tumor growth. In this model, we observed strong antitumor activity against EG7 but not EL4 in TOG-immunized B6 mice but not in J $\alpha 18^{-/-}$mice (Figure 3F). The growth of EG7 tumor cells in J $\alpha 18^{-/-}$mice was significantly suppressed by adoptive transfer of the $7 \mathrm{a}-\mathrm{NKT}$ or $7 \mathrm{~g}$-NKT cells, whereas the growth of EL4 was not. These results indicate that the iPSC-derived NKT cells can enhance the generation of functionally sufficient numbers of antigen-specific CD8 T cells to exert antitumor immunity. Therefore, the iPSC-derived NKT cells are functionally competent to mediate adjuvant activity in vivo.

Generation of iPSC from splenic NKT cells. We finally confirmed splenic NKT cells also could be reprogrammed into iPSCs. NKT cells $\left(1 \times 10^{6}\right)$ were isolated from normal B6 splenocytes and then activated by anti-CD3/CD28 magnetic beads together with IL-12 $(10 \mathrm{ng} / \mathrm{ml})$ and IL-2 $(10 \mathrm{ng} / \mathrm{ml})$ for 1 week, followed by reprogramming according to the conventional protocol (14). From 2 experiments, we established at least 3 independent iPSC-like lines. After confirming that the $14 \mathrm{k}$ cell line (iPSC-14k) was NKT cell-derived, based on the nucleotide and deduced amino acid sequences of the CDR3 junctional regions of the Tcra and Tcrb loci (Figure 4A), we focused on this line to determine whether it is an authentic iPSC. Immunofluorescent analysis revealed the expression of SSEA1, Oct3/4, and Nanog in the iPSC-14k cells (Figure 4B) and the expression of endogenous Oct3/4, Sox2, Klf4, Nanog, Ecat1, Gdf3, Rex1, and Zfp296 mRNA could be detected by RT-PCR (Figure 4C). Genome-wide gene expression profiles of the iPSC-14k cells showed a higher correlation coefficient to ESCs or iPSCs, such as NKT-ES C-1 and iPSC-7a or iPSC-7g, than to splenic NKT cells (Figure 4D and Supplemental Figure 9A). Therefore, the iPSC-14k cells were shown to be adequately reprogrammed. We went on to test the ability of these cells to form teratomas and chimeras as described above. Teratomas with 3 germ layers were generated from the iPSC-14k cells upon s.c. injection into nude mice (Supplemental Figure 9B). Three coat color chimeras were generated upon injection into BALB/c blastocysts, and one of them was a germline chimera (Figure 4E). Taken together, the iPSC-14k cells were confirmed to be an authentic iPSC line.

We further examined the redifferentiation potential of the iPSC-14k cells into splenic NKT cells in vitro in the 25-day culture system. In the culture system, the iPSC-14k cells $\left(1 \times 10^{5}\right)$ gave rise to $3 \times 10^{7}$ $\alpha$-GalCer/CD1d dimer ${ }^{+}$TCR- $\beta^{+}$cells, equivalent to the potential of iPSC-7a or iPSC-7g cells. The induced NKT cells were mostly immature $\mathrm{CD}^{+} \mathrm{CD}^{+}$phenotypes (Figure $4 \mathrm{~F}$ ) with $\mathrm{CD} 24^{\text {hi }}, \mathrm{CD} 44^{\text {lo }}$, $\mathrm{CD}^{2} \mathrm{~L}^{\text {hi }}, \mathrm{CD} 122^{\mathrm{lo}}, \mathrm{CD}^{\mathrm{l}} 9^{\mathrm{lo}}$, and NKG2D- (Supplemental Figure 5), and of note, approximately $30 \%$ of them expressed NK1.1 (Figure $4 \mathrm{~F})$. $\alpha$-GalCer/CD1d dimer ${ }^{+}$TCR- $\beta^{+}$cells derived from the iPSC- $14 \mathrm{k}$ also exhibited a significant proliferative response and production of IFN- $\gamma$ and IL-4 upon antigenic stimuli (Figure 4G). Therefore, the iPSC-14k-derived $\alpha$-GalCer/CD1d dimer ${ }^{+}$TCR- $\beta^{+}$cells represent NKT cells. However, since some of the cells express NK1.1, their developmental stage may be slightly more advanced compared with those derived from the iPSC-7a or iPSC-7g cells. It is interesting 
A

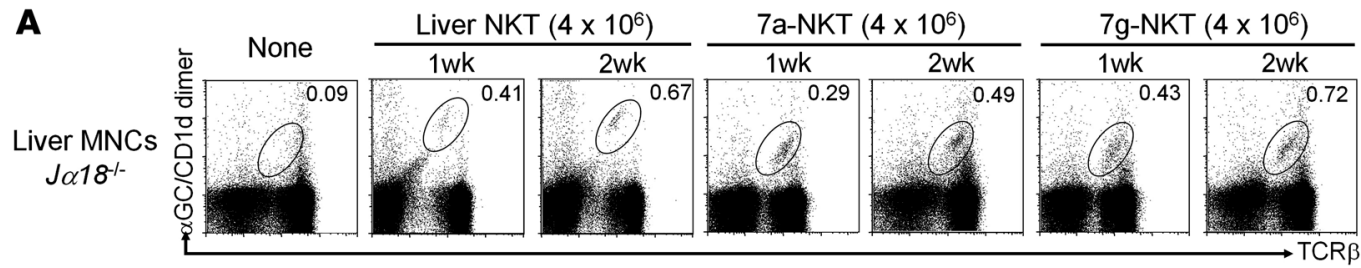

B

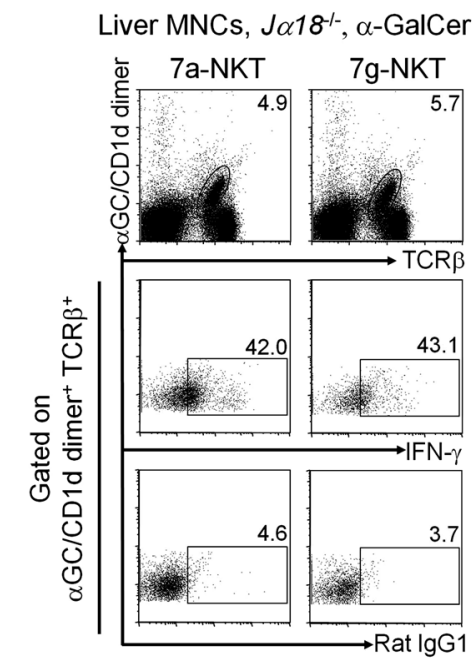

C Liver MNCs, $J \alpha 18^{-/}, \alpha$-GalCer i.v.

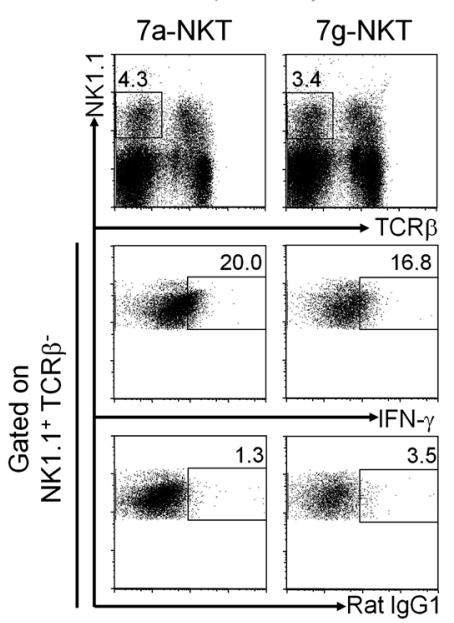

D

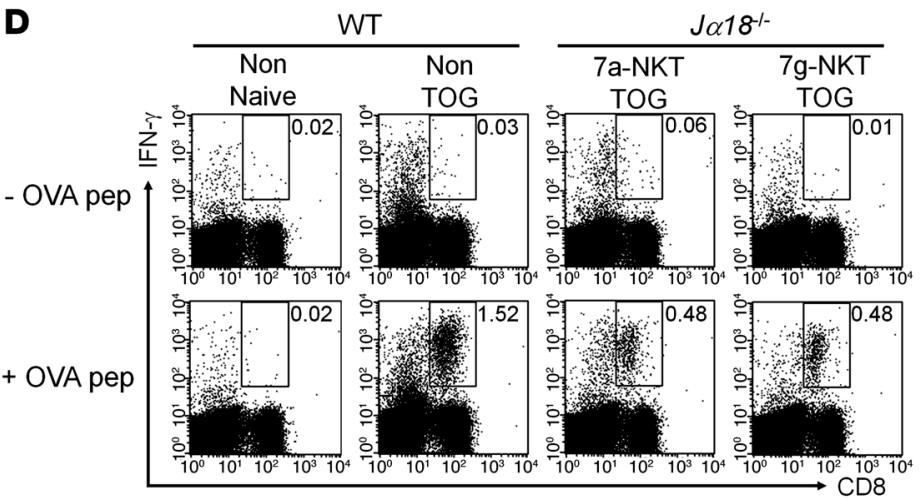

E

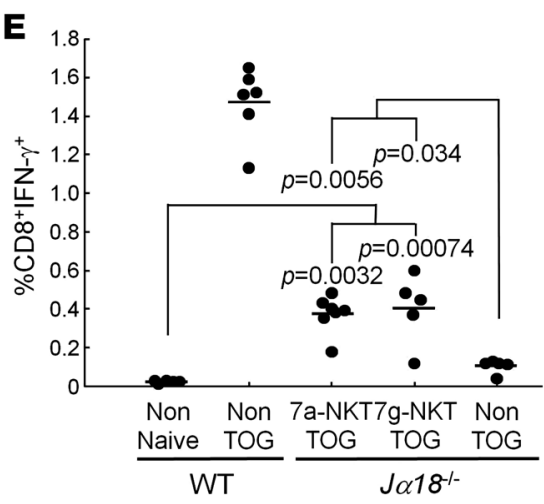

$\mathbf{F}$

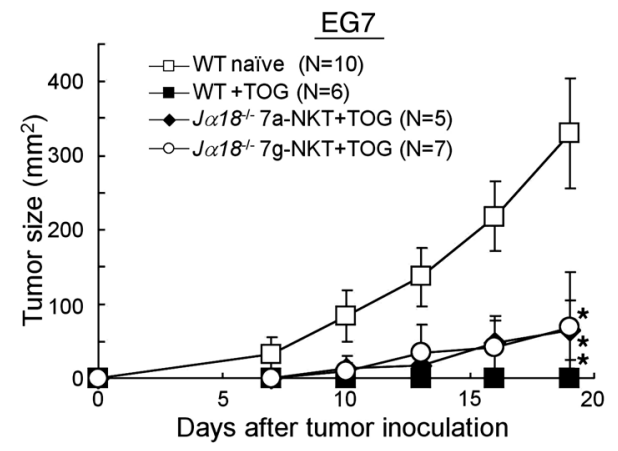

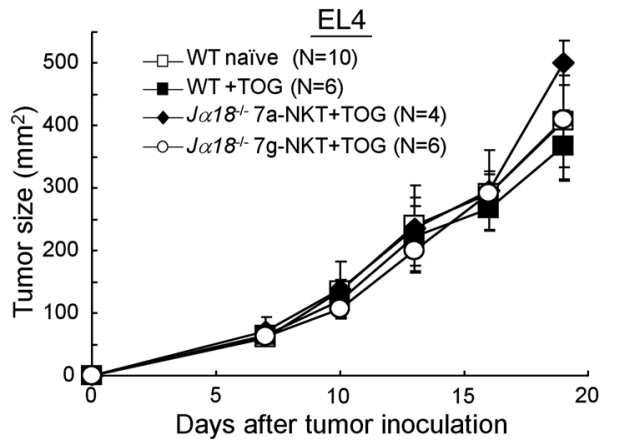




\section{Figure 3}

Adjuvant activity of iPSC-derived NKT cells on antitumor responses in

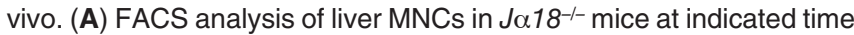
points after adoptive transfer of liver NKT, 7a-NKT, or 7g-NKT cells $\left(4 \times 10^{6} \mathrm{cell} /\right.$ mouse). (B and $\left.\mathbf{C}\right)$ In vivo expansion and IFN- $\gamma$ production of 7a-NKT and 7g-NKT cells $(B)$ and their bystander effects on NK

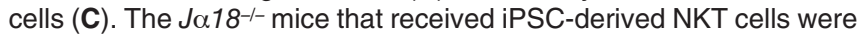
primed i.v. with $\alpha$-GalCer $(2 \mu \mathrm{g})$. The indicated cells in liver MNCs in $\mathbf{B}$ and $\mathbf{C}$ were investigated for their intracellular IFN- $\gamma$ production by FACS using anti-IFN- $\gamma$ compared with rat IgG1. (D-F) iPSC-derived NKT cell-mediated bystander adjuvant activity on CD8 T cells ( $D$ and E) and antitumor responses (F) based on the experimental procedure shown in Supplemental Figure 7. (D) IFN- $\gamma$ production by CD8 T cells upon restimulation with $\mathrm{OVA}_{257-264}$ peptide in vitro. Representative data from 3 experiments are shown. Numbers show percentage of each gate. (E) Summary and statistical analysis for the results shown in $\mathbf{D}$. The frequency of OVA-specific, IFN- $\gamma$-producing CD8 T cells is summarized as percentage of total lymphocytes in the spleen. $P$ values indicate differences between each group. (F) Adjuvant activity of 7a-NKT and 7g-NKT cells on antitumor responses. Mice were challenged with $2 \times 10^{5}$ EL4- or OVA-expressing EL4 (EG7) cells s.c. Tumor size in each group was measured at the indicated time points. Mean \pm SD from 2 independent experiments is shown. ${ }^{*} P<0.01$, differences between WT naive and other groups in EG7-injected mice.

that the ability of iPSC-14k-derived NKT cells to proliferate and to produce IFN- $\gamma$ was similar to that of iPS-7a-derived NKT cells or splenic NKT cells in the 25-day culture (Figure 2), while no significant responses were obtained by iPSC-14k-derived NKT cells generated in the 20-day culture system (Figure 4G), indicating that the functions of iPSC-derived NKT cells are largely dependent on the duration of the culture in vitro and that at least the 25-day culture period is required to obtain functional iPSC-derived NKT cells.

\section{Discussion}

Although several attempts have been made to generate iPSCs from mature B or T lymphocyte cells by the introduction of Oct3/4, Sox2, $K l f 4$, and $c-M y c$, the efficiency is very low (25). Recently, B- or T-derived iPSCs have efficiently been generated by gene modulation of the myeloid transcription factor CCAAT/enhancer-binding protein- $\alpha$ (C/EBP $\alpha$ ) or Pax5 for B cells (26), or of the p53/p21 pathway for $\mathrm{T}$ cells (27). However, efforts to regenerate functional B or T lymphocytes from these lymphocyte-derived iPSCs have remained unsuccessful. In the present studies, we have successfully demonstrated for what we believe is the first time the generation of functional NKT cells in vitro from splenic NKT cell-derived iPSCs and also the clinical potential of iPSC-derived NKT cells to control the growth of a syngeneic tumor through their adjuvant activity. Given the promise of patient-specific therapy, it might be prudent to consider the establishment of cell banks of NKT cell-derived iPSCs. Since the functions and antigen specificity of NKT cells are highly conserved between mice and humans, this study has opened up an alternative pathway to realize the potential of NKT cell-targeted therapy in cancer patients. Moreover, NKT cells play multiple roles in modulation of immune responses, such as in antiviral immunity and rejection of transplanted tissues $(1,4)$. Therefore, therapeutic applications of iPSC-derived NKT cells through their adjuvant activity could be extended to other serious diseases involving immune mechanisms.

Furthermore, this and our previous studies have suggested that derivation of NKT cells from iPSCs does not depend on the cellular origin of the iPSCs but likely on their intrinsic ability to express the invariant TCR that is specific for NKT cells. If this interpretation is correct, it would imply that iPSCs and ESCs could be induced to differentiate into the NKT cell lineage once the appropriate rearranged Tor genes were introduced, either by homologous recombination or simple transfection. Since this approach should be more generally available than the more complex procedure of generating iPSCs from NKT cells by retrovirus-mediated reprogramming, we are currently addressing this possibility in mice.

During the course of this study, we found some subtle differences among iPSC-derived NKT cell lines in their phenotypes and in their ability to proliferate or to produce cytokines (Figure 2 and Supplemental Figure 6). Such functional heterogeneity that is manifested upon differentiation has previously been reported for human ESCs (28). However, in general, there is no significant difference in their phenotypes (Supplemental Figures 5 and 8). The phenotypic changes are largely dependent on culture conditions. In the 20-day culture system, iPSCs cultured on OP9/Dll-1 give rise to NKT cells bearing immature phenotypes similar to $\mathrm{CD} 4^{+} \mathrm{CD} 8^{+} \mathrm{DP}$ thymocytes in vitro as shown in Figure 2, while iPSC-derived NKT cells in the switch culture showed mature $\mathrm{CD}^{+}$or double-negative (DN) NKT cell phenotypes (Supplemental Figure 3B). These are similar to those obtained by the ESC culture as described (13). Therefore, Notch signaling inhibits phenotypic maturation of NKT cells.

The phenotypic maturation of iPSC-derived NKT cells with immature phenotypes occurs in vivo because the majority of iPSC-derived NKT cells, when transferred into J $\alpha 18^{-/-}$mice in vivo, developed downmodulated expression of CD24 and CD62L but augmented expression of CD44, CD69, CD122, and NKG2D (Supplemental Figure 8), similar to mature liver NKT cell phenotypes.

Most important is that, without maturation of cell surface phenotypes, iPSC-derived NKT cells acquire their potential to produce large amounts of IFN- $\gamma$, which is essential for in vivo adjuvant activity on antitumor responses. In fact, the iPSC-derived NKT cells obtained in the 25-day culture showed the ability to produce cytokines at levels similar to those of splenic NKT cells (Figure 2D and Figure $4 \mathrm{G}$ ). In particular, IFN- $\gamma$ production was significantly high in the 25-day culture system using OP9/Dll-1 (Figure 4G and Figure 2D), although, similar to ESC-derived NKT cells (13), iPSCderived NKT cells mainly produced IL-4 but not IFN- $\gamma$ in the 20-day culture system using OP9/Dll-1 (Supplemental Figure 3C). Therefore, IFN- $\gamma$ production by iPSC-derived NKT cells is apparently due to the duration of the culture. In fact, iPSC-derived NKT cells produced large amounts of IFN- $\gamma$ at culture day 25 , but produced small amounts of IFN- $\gamma$ at day 20 (see Figure 4G). Thus, immature cell surface phenotypes on iPSC-derived NKT cells generated in the present system do not correlate with their functional activity.

Taken collectively, the 25-day culture system using IL-7 and Flt3L and OP9/Dll-1 has several advantages; it yielded 10 times the number of NKT cells and produced 5 times the amount of IFN- $\gamma$ (Figure 2D and Figure 4G) than what was obtained in the 20-day culture system (Supplemental Figure 3). Although the system we described here is a mouse model, it is helpful to establish methods for generation of iPSCs from human NKT cells and protocols for developing NKT cells from iPSCs suitable for clinical settings.

\section{Methods}

Cells. The cells used in the present studies were ESCs (R1, E14); cloned ESCs, such as NKT-ES C-1 and NKT-ES D-1, obtained by direct nuclear transfer of B6 splenic NKT cells (13); MEFs (NKT-MEF) obtained from NKT B6 mice generated by the cloned ESCs; iPSCs (iPSC-7a, iPSC-7g) gen- 
A

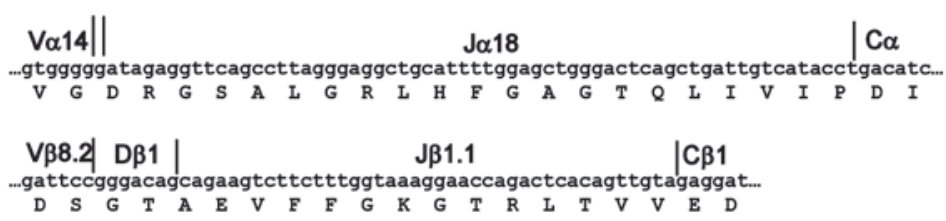

C

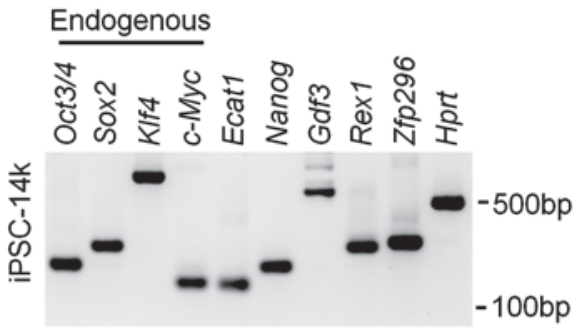

B

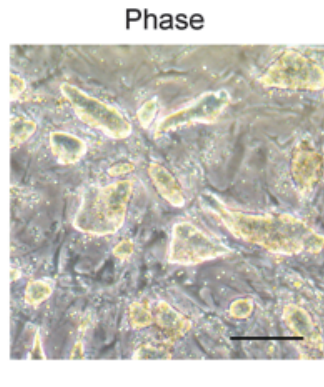

Oct3/4 DAPI

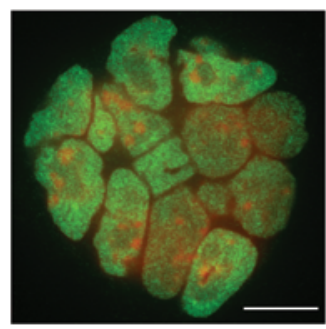

SSEA1 DAPI

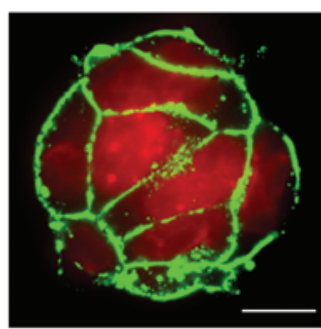

Nanog DAPI

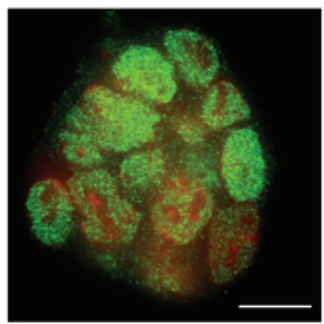

D

\begin{tabular}{|c|c|c|c|c|c|}
\hline & iPSC-14k & NKT-ES C-1 & iPSC-7a & iPSC-7g & splenic NKT1 splenic NKT2 \\
\hline splenic NKT2 & 0.822 & 0.833 & 0.868 & 0.899 & 0.892 \\
\hline splenic NKT1 & 0.794 & 0.787 & 0.835 & 0.847 & 1.000 \\
\hline iPSC-7g & 0.901 & 0.924 & 0.933 & 1.000 & \\
\hline iPSC-7a & 0.905 & 0.925 & 1.000 & & \\
\hline NKT-ES C-1 & $\mathbf{0 . 9 5 9}$ & 1.000 & & & \\
\hline iPSC-14k & 1.000 & & & &
\end{tabular}

E

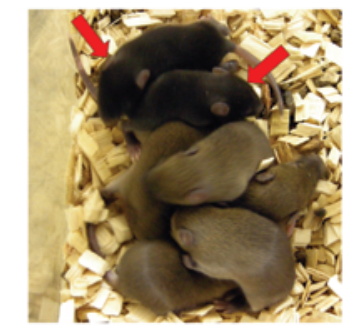

$\mathbf{F}$

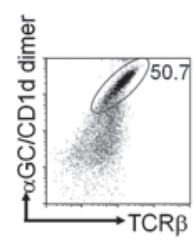

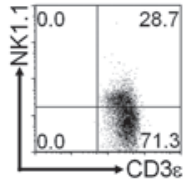

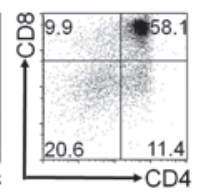

G

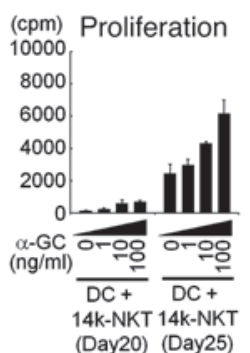

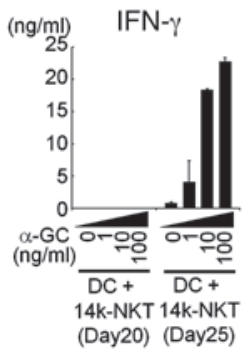

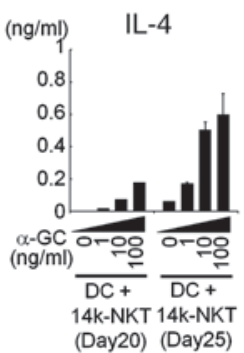

\section{Figure 4}

Generation of iPSC from splenic NKT cells and their development of iPSC-derived NKT cells with function in vitro. (A) Nucleotide and deduced amino acid sequences of the CDR3 junctional regions of the Tcra and Tcrb loci in iPSC-14k cells. (B) Colony shape and expression of SSEA1, Oct3/4, and Nanog in iPSC-14k. Scale bars: 100 um (phase); $10 \mu \mathrm{m}$ (immunofluorescence). (C) Expression of ESC marker genes in iPSC-14k cells analyzed by RT-PCR. (D) Analysis of global gene expression profiles comparing iPSC-14k cells to NKT-ES C-1, iPSC-7a, iPSC-7g, and splenic NKT cells. Note that the iPSC-14k cells are closely related to NKT-ES C-1, iPSC-7a, and iPSC-7g. (E) Offspring obtained by mating chimeras generated from the iPSC-14k cells and C57BL/6 females. Black coat color (indicated by arrows) represents germline transmission of iPSC-14k. (F) Expression of cell surface markers on the 14k-NKT cells. Numbers show percentage of each gate. (G) Proliferative response (left) and cytokine production (middle and right) by $14 \mathrm{k}-\mathrm{NKT}$ cells (culture day 20 and day 25) upon stimulation with $\alpha$-GalCer. Mean \pm SD of triplicate wells is shown. One representative experiment of 3 is shown.

erated by NKT-MEF or iPSCs (iPSC-14k) by B6 splenic NKT cells; iPSCderived NKT cells (7a-NKT, 7g-NKT, 14k-NKT); and splenic NKT cells.

Generation of $i P S C s$. NKT-MEF cells were reprogrammed by using retroviral vectors expressing Oct3/4, Sox2, Klf4, and Nanog according to the protocol established previously (14), which generated iPSC clones, such as iPSC-7a and iPSC-7g. The iPSCs from splenic NKT cell lines, such as iPSC-14k, were established by Yamanaka's method (14) using B6 splenic NKT cells $\left(1 \times 10^{6}\right)$ activated with anti-CD3 and anti-CD28 in the presence of IL-12 and IL-2.
Nucleotide sequences for primer pairs used for RT-PCR analysis of ESC markers are available in a previous report by Takahashi and Yamanaka (14). All animal studies were approved by the RIKEN Yokohama Institute Review Board.

Genomic PCR. Genomic DNA was isolated from cells using a Gentra Puregene kit (QIAGEN) for genotyping. Genomic PCR was performed with LA Taq (Takara). Primers used are listed in Supplemental Table 1.

Immunofluorescence. Immunofluorescence staining was performed using the following primary antibodies: anti-SSEA-1 (MAB4301; Milli- 
pore), anti-Nanog (ab21603; Abcam), and anti-Oct3/4 (sc-5279; Santa Cruz Biotechnology Inc.).

Flow cytometry. FITC-, PE-, PE-Cy7-, APC-, APC-Cy7-, or Pacific Blueconjugated $\mathrm{mAbs}$ were purchased from $\mathrm{BD}$ Biosciences or eBioscience and are listed in Supplemental Table 2. Flow cytometry was performed using FACSCalibur and FACSAria (BD) with FlowJo software (Tree Star). The purity of sorted cells was usually greater than $99 \%$.

Bisulfite DNA sequencing analysis. Genomic DNA was purified from ESCs, iPSCs, and MEFs by using the Allprep DNA/RNA Mini Kit (QIAGEN), and $2 \mu \mathrm{g}$ of DNA was utilized for bisulfite conversion by using the EpiTect Bisulfite Kit (QIAGEN), according to the supplier's protocol. Bisulfite-treated DNA was amplified by PCR and cloned into the PCR 2.1-TOPO vectors (Invitrogen); positive clones were examined by DNA sequencing using the BigDye Terminator v3.1 Cycle Sequencing Kit (ABI), following the vendor's instructions. Sequences extracted from individual clones were then analyzed to reveal the status of $\mathrm{CPG}$ methylation for the Oct3/4 and Nanog loci (15).

Correlation analysis of microarrays. Gene expression detected by using microarrays was normalized by the quantile normalization method (29). Pearson's correlation values of logarithms of all signal intensities from 45,101 probes were calculated, and we performed hierarchal clustering of correlation matrices to indicate the degree of similarity between cell types. Scatter diagrams (Supplemental Figure 2) were drawn to display how similarly or differently genes were expressed in 2 samples. These diagrams contain only probes whose signals were present.

Generation of NKT cells from iPSCs. OP9/control and OP9/Dll-1 were used as a monolayer with OP9 medium ( $\alpha$-MEM supplemented with $20 \%$ FCS [HyClone], $10 \mathrm{U} / \mathrm{ml}$ penicillin, $100 \mu \mathrm{g} / \mathrm{ml}$ streptomycin, and $2.2 \mathrm{~g} / \mathrm{l}$ of sodium bicarbonate). In brief, iPSCs (iPSC-7a, iPSC-7g, or iPSC-14k; $5 \times 10^{4}$ ) were cultured with OP9/Dll-1 as an adherent cell layer in OP9 medium. On day 6 of culture, most iPSC colonies were disrupted by treatment with $0.25 \%$ trypsin (Invitrogen). The nonadherent cells were then cultured for another 4 days on a fresh OP9/Dll-1 in OP9 medium with the addition of Flt3L (10 ng/ml; Peprotech). On day 10 of culture and every 4 days thereafter, nonadherent iPSCderived hematopoietic cells were cultured on a fresh OP9/Dll-1 in OP9 medium with Flt3L (10 ng/ml; Peprotech) and IL-7 (1 ng/ml; Peprotech). In some experiments, cells obtained at the indicated days of the NKT-ES/OP9/Dll-1 cultures were further cocultured on OP9/control. The method outlined above is similar to a published protocol (also see Supplemental Figure 3) (30).

Quantitative real-time PCR. Total RNA was isolated from FACS-purified cell populations using the TRIzOL reagent (Invitrogen). cDNA was prepared by Superscript III RNase $\mathrm{H}^{-}$Reverse Transcriptase with random hexamers (Invitrogen). Quantitative real-time PCR was performed with SYBR GreenER qPCR SuperMix (Invitrogen) for ABI PRISM 7900HT (Applied Biosystems). Cells $\left(2 \times 10^{3}\right)$ for Zbtb16, Sh2d1a, or Hprt were used as templates. Gene-specific primer sequences were as follows: Zbtb16-fw: AACGGTTCCTGGACAGTTTG, Zbtb16-rv: CCCACACAGCAGACAGAAGA; Sh2d1a-fw: ACGCCTCTGCAGTATCCAGT, Sh2d1a-rv: ATGGTGCATTCAGGCAGATA; and Hprt-fw: TCCTCCTCAGACCGCTTTT, Hprt-rv: CCTGGTTCATCATCGCTAATC. Quantitative analysis was performed by $\Delta \Delta C$ method by using hprt as an internal control.

Intracellular cytokine staining. Intracellular cytokine staining was performed as described previously (31). For IFN- $\gamma$ staining in NKT cells and
NK cells, liver MNCs from J $\alpha 18^{-/-}$mice that had received iPSC-derived NKT cells were prepared 16 hours after administration of $\alpha$-GalCer. Brefeldin A (Sigma-Aldrich) was added for the last 4 to 5 hours of culture of liver MNCs to accumulate intracellular cytokines. Cells were then washed and incubated with $\alpha$-GalCer/CD1d dimer and anti-mouse TCR- $\beta$ mAb for NKT cells and anti-mouse NK1.1 and anti-mouse TCR- $\beta$ $\mathrm{mAb}$ for NK cells for 20 minutes at $4{ }^{\circ} \mathrm{C}$ after first blocking $\mathrm{Fc}$ receptors with an anti-CD16/CD32 antibody. Following fixation with Cytofix/ Cytoperm Plus (BD Biosciences), cells were stained for intracellular IFN- $\gamma$ for 15 minutes at room temperature.

Tcell immunity. The experimental protocols for evaluating $\mathrm{T}$ cell immunity have been previously described in detail (see also Supplemental Figure 7 and ref. 5). Briefly, to prepare the cell-associated OVA, spleen cells from $T A P^{-1-}$ mice were treated with hypertonic medium in the presence of $10 \mathrm{mg} / \mathrm{ml}$ OVA and then incubated with hypotonic medium. The cellassociated form of OVA $\left(2 \times 10^{7}\right.$ cells/mouse $)$ and $2 \mu \mathrm{g} \alpha$-GalCer were coadministered i.v. to mice. Seven days later, spleen cells from immunized mice were cultured for 6 hours with $1 \mu \mathrm{M}_{\text {OVA }}$ 257-264 peptide in the presence of brefeldin A to accumulate IFN- $\gamma$ intracellularly. Cells were incubated with the $2.4 \mathrm{G} 2 \mathrm{mAb}$, then FITC anti-CD8 for 20 minutes at room temperature, and then permeabilized and stained for PE-conjugated anti-IFN- $\gamma$ (XMG1.2) mAb. For reconstitution in J $\alpha 18^{-/-}$mice, iPSCderived NKT cells (7a-NKT or $7 \mathrm{~g}-\mathrm{NKT}$ cells; $4 \times 10^{6}$ cells per mouse) were transferred i.v. Two weeks later, the mice were immunized with cell-associated OVA together with $\alpha$-GalCer (TOG). A week later, spleen cells from the recipient mice were challenged with $\mathrm{OVA}_{257-264}$ peptide and analyzed for IFN- $\gamma$ production as above.

Tumor protection experiments. As above, $2 \times 10^{7}$ OVA-loaded spleen cells and $2 \mu \mathrm{g} \alpha$-GalCer were coadministered to WT or $J \alpha 18^{-/-}$mice that had been transferred with 7a-NKT or 7g-NKT cells 2 weeks before. A week later, $2 \times 10^{5}$ EG7 cells were inoculated s.c. into the TOG-treated or naive mice. The parental non-OVA-transduced EL4 cells were used as control tumor cells. Tumor growth was monitored by measuring tumor size.

Statistics. The statistical significance of differences between the experimental groups was determined by the Mann-Whitney exact rank sum test. $P<0.05$ was considered statistically significant.

\section{Acknowledgments}

The authors are grateful to P.D. Burrows for helpful comments and constructive criticism in the preparation of the manuscript.

Received for publication December 14, 2009, and accepted in revised form April 14, 2010.

Address correspondence to: Haruhiko Koseki, Laboratory for Developmental Genetics, RIKEN Research Center for Allergy and Immunology, Yokohama, Kanagawa 230-0045, Japan. Phone: 81.45.503.9689; Fax: 81.45.503.9688; E-mail: koseki@rcai.riken.jp. Or to: Masaru Taniguchi, Laboratory for Immune Regulation, RIKEN Research Center for Allergy and Immunology, Yokohama, Kanagawa 230-0045, Japan. Phone: 81.45.503.7001; Fax: 81.45.503.7003; E-mail: taniguti@rcai.riken.jp.
1. Taniguchi M, Harada M, Kojo S, Nakayama T, Wakao $H$. The regulatory role of Valpha14 NKT cells in innate and acquired immune response. Annu Rev Immunol. 2003;21:483-513.

2. Lantz O, Bendelac A. An invariant T cell receptor alpha chain is used by a unique subset of major histocompatibility complex class I-specific CD $4^{+}$

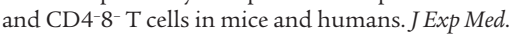
1994;180(3):1097-1106.
3. Exley M, Garcia J, Balk SP, Porcelli S. Requirements for CD1d recognition by human invariant Valpha24+ CD4-CD8- T cells. J Exp Med. 1997;186(1):109-120.

4. Fujii S, Shimizu K, Hemmi H, Steinman RM. Innate Valpha14(+) natural killer T cells mature dendritic cells, leading to strong adaptive immunity. Immunol Rev. 2007;220:183-198.

5. Cui J, et al. Requirement for Valpha14 NKT cells in
IL-12-mediated rejection of tumors. Science. 1997; 278(5343):1623-1626.

6. Terabe M, Berzofsky JA. The role of NKT cells in tumor immunity. Adv Cancer Res. 2008;101:277-348.

7. Dhodapkar MV. Harnessing human CD1d restricted T cells for tumor immunity: progress and challenges. Front Biosci. 2009;14:796-807.

8. Okai M, et al. Human peripheral blood Valpha24+ Vbeta11+ NKT cells expand following administra- 
tion of alpha-galactosylceramide-pulsed dendritic cells. Vox Sang. 2002;83(3):250-253.

9. Nieda M, et al. Therapeutic activation of Valpha24 $4^{+}$beta $11^{+}$NKT cells in human subjects results in highly coordinated secondary activation of acquired and innate immunity. Blood. 2004; 103(2):383-389.

10. Chang DH, et al. Sustained expansion of NKT cells and antigen-specific $T$ cells after injection of alphagalactosyl-ceramide loaded mature dendritic cells in cancer patients. J Exp Med. 2005;201(9):1503-1517.

11. Motohashi S, et al. A phase I-II study of alphagalactosylceramide-pulsed IL-2/GM-CSF-cultured peripheral blood mononuclear cells in patients with advanced and recurrent non-small cell lung cancer. J Immunol. 2009;182(4):2492-2501.

12. Motohashi S, et al. A phase I study of in vitro expanded natural killer $\mathrm{T}$ cells in patients with advanced and recurrent non-small cell lung cancer. Clin Cancer Res. 2006;12(20 pt 1):6079-6086.

13. Watarai $\mathrm{H}$, et al. Generation of functional NKT cells in vitro from embryonic stem cells bearing rearranged invariant V\{alpha\}14-J\{alpha\}18 TCR\{alpha\} gene. Blood. 2010;115(2):230-237.

14. Takahashi K, Yamanaka S. Induction of pluripotent stem cells from mouse embryonic and adult fibroblast cultures by defined factors. Cell. 2006;126(4):663-676.

15. Okita K, Ichisaka T, Yamanaka S. Generation of germline-competent induced pluripotent stem cells. Nature. 2007;448(7151):313-317.

16 . Wernig $\mathrm{M}$, et al. In vitro reprogramming of fibroblasts into a pluripotent ES-cell-like state. Nature. 2007:448(7151):318-324

17. Savagem AK, et al. The transcription factor PLZF directs the effector program of the NKT cell lineage. Immunity. 2008;29(3):391-403.

18. Kovalovsky D, et al. The BTB-zinc finger transcriptional regulator PLZF controls the development of invariant natural killer $\mathrm{T}$ cell effector functions. Nat Immunol. 2008;9(9):1055-1064.

19. Nichols KE, et al. Regulation of NKT cell development by SAP, the protein defective in XLP. Nat Med. 2005;11(3):340-345.

20. Pasquier B, et al. Defective NKT cell development in mice and humans lacking the adapter SAP, the $\mathrm{X}$-linked lymphoproliferative syndrome gene product. J Exp Med. 2005;201(5):695-701.

21. Gonzalez-Aseguinolaza G, et al. Natural killer $T$ cell ligand alpha-galactosylceramide enhances protective immunity induced by malaria vaccines. J Exp Med. 2002;195(5):617-624.

22. Fujii S, Shimizu K, Smith C, Bonifaz L, Steinman RM. Activation of natural killer T cells by alphagalactosylceramide rapidly induces the full maturation of dendritic cells in vivo and thereby acts as an adjuvant for combined CD4 and CD8 T cell immunity to a coadministered protein. J Exp Med. 2003;198(2):267-279.

23. Hermans IF, et al. NKT cells enhance CD4+ and
CD8 $+\mathrm{T}$ cell responses to soluble antigen in vivo through direct interaction with dendritic cells. JImmunol. 2003;171(10):5140-5147.

24. Fujii S, et al. Glycolipid alpha-C-galactosylceramide is a distinct inducer of dendritic cell function during innate and adaptive immune responses of mice. Proc Natl Acad Sci U S A. 2006;103(30):11252-11257.

25. Yamanaka S. Strategies and new developments in the generation of patient-specific pluripotent stem cells. Cell Stem Cell. 2007;1(1):39-49.

26. Hanna J, et al. Direct reprogramming of terminally differentiated mature B lymphocytes to pluripotency. Cell. 2008;133(2):250-264.

27. Hong $\mathrm{H}$, et al. Suppression of induced pluripotent stem cell generation by the p53-p21 pathway. Nature. 2009;460(7259):1132-1135.

28. Osafune K, et al. Marked differences in differentiation propensity among human embryonic stem cell lines. Nat Biotechnol. 2008;26(3):313-315.

29. Bolstad BM, Collin F, Simpson KM, Irizarry RA, Speed TP. Experimental design and low-level analysis of microarray data. Int Rev Neurobiol. 2004; 60:25-58.

30. Cho SK, Zúñiga-Pflücker JC. Development of lymphoid lineages from embryonic stem cells in vitro. Methods Enzymol. 2003;365:158-169.

31. Watarai H, Nakagawa R, Omori-Miyake M, Dashtsoodol N, Taniguchi M. Methods for detection, isolation and culture of mouse and human invariant NKT cells. Nat Protoc. 2008;3(1):70-78. 